\title{
Evaluation of small-diameter silk vascular grafts implanted in dogs
}

Takashi Tanaka, DVM, PhD, ${ }^{a}$ Ryo Tanaka, DVM, PhD, ${ }^{a}$ Yoko Ogawa, BS,,${ }^{\mathrm{b}}$ Yoshihide Takagi, BS, ${ }^{\mathrm{b}}$ Masataka Sata, MD, $\mathrm{PhD},{ }^{\mathrm{c}}$ and Tetsuo Asakura, $\mathrm{PhD}^{\mathrm{d}}$

\section{ABSTRACT}

Objectives: Vascular replacement is one treatment for cardiovascular disease. However, in blood vessels with a diameter less than $6 \mathrm{~mm}$, the existing artificial vascular grafts may be occluded by thrombus formation or intimal hyperplasia. Thus, new artificial vascular grafts need to be developed. We have developed a small-diameter artificial vascular graft made of silk fibroin. The implantation of such graft has been evaluated mainly in rats. However, only a few reports describe long-term implantation in large animal models. Therefore, modified silk fibroin artificial vascular grafts were implanted in the femoral arteries of dogs, and their patency and remodeling ability were investigated.

Methods: Six beagles weighing 10 to $12 \mathrm{~kg}$ were used for the in vivo study. Grafts (4 cm length $\times 3.5 \mathrm{~mm}$ inner diameter) were implanted in the femoral artery of 6 dogs. The patency of the graft was monitored using vascular ultrasound apparatus. At 3 months', 5 months', and 1-year postimplantation, the graft was retrieved and conducted histopathologic examination.

Results: No side effects, such as ischemia, paralysis, and edema of the hind legs, were observed postimplantation. Five of the 6 grafts exhibited a high patency rate, and the lumen was covered with vascular endothelial cells in the central part of the graft 3 months' postimplantation.

Conclusions: Based on these results, artificial silk fibroin vascular grafts implanted in the femoral arteries of dogs exhibit high patency and remodeling ability. Silk fibroin grafts may be clinically applicable as an artificial vascular graft in smalldiameter <6 mm. (JTCVS Open 2021;6:148-56)

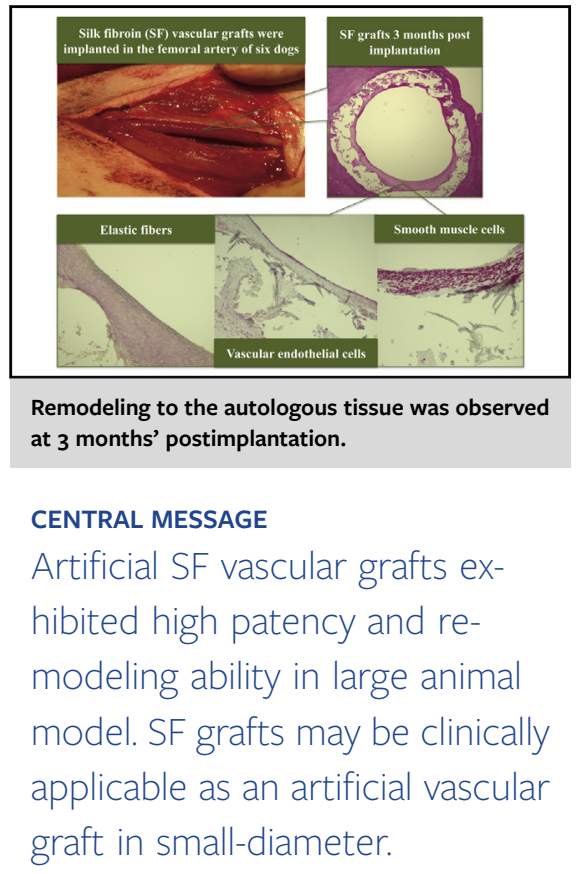

\section{PERSPECTIVE}

For the clinical application of SF artificial vascular grafts, it was important to evaluate the implantation in larger animals. In this study, artificial SF vascular grafts implanted in the femoral arteries of dogs exhibit high patency and remodeling ability. SF grafts may be clinically applicable as an artificial vascular graft in small-diameter $<6 \mathrm{~mm}$.

See Commentaries on pages 157 and 159.

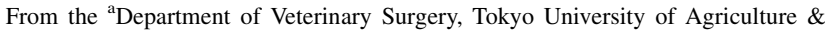
Technology, Fuchu, Tokyo; ${ }^{b}$ Fukui Wrap Knitting Co., Ltd, Nishikaihatsu, Fukui; ${ }^{c}$ Department of Cardiovascular Medicine, Tokushima University Graduate School of Biomedical Sciences, Tokushima; and ${ }^{\mathrm{d} D e p a r t m e n t ~ o f ~ B i o t e c h n o l o g y, ~ T o k y o ~}$ University of Agriculture \& Technology, Koganei, Tokyo, Japan.

This work was supported by JSPS KAKENHI, Grant-in-Aid for Scientific Research, Grant Number JP26248050, JP19K05609 and JP20K12643.

Received for publication Feb 23, 2021; accepted for publication Feb 26, 2021; available ahead of print March 30, 2021.

Address for reprints: Takashi Tanaka, DVM, PhD, Department of Veterinary Surgery, Tokyo University of Agriculture \& Technology, Fuchu, Tokyo, 183-8590 Japan (E-mail: bamse.rizea.vicky.polta@gmail.com).

2666-2736

Copyright (c) 2021 The Authors. Published by Elsevier Inc. on behalf of The American Association for Thoracic Surgery. This is an open access article under the CC BY-NC-

ND license (http://creativecommons.org/licenses/by-nc-nd/4.0/).

https://doi.org/10.1016/j.xjon.2021.02.008
The incidence of cardiovascular diseases has increased in recent years. Therefore, there has been an increasing need for the development of small-diameter artificial vascular grafts worldwide. ${ }^{1}$ Synthetic polymers, such as expanded polytetrafluoroethylene (ePTFE) and poly(ethylene terephthalate), are used to create artificial vascular grafts with diameters $>6 \mathrm{~mm} .{ }^{2,3}$ However, occlusion due to thrombus formation and intimal hyperplasia occurs in these vascular grafts. Occlusion and thrombus formation are caused by the lack of vascular endothelial cells and mismatch with endogenous vessels. ${ }^{4-6}$ Thus, small-diameter artificial blood vessels using scaffolding materials that promote remodeling with endogenous cells are being investigated. ${ }^{7,8}$ 


\section{Abbreviations and Acronyms}

ePTFE $=$ expanded polytetrafluoroethylene

$\mathrm{SF}=$ silk fibroin

However, none of the new artificial vascular grafts have been clinically used as of yet.

Various materials to replace malfunctioning or diseased cardiovascular tissues have been investigated. ${ }^{9}$ Degradable synthetic materials, such as polyglycolic acid, poly-L-lactic acid, poly(lactide-co-glycolide), and polycaprolactone, have been investigated and yielded promising results. ${ }^{10}$ However, none of these materials are suitable for the generation of small-diameter vascular grafts due to thrombus formation and compliance mismatch. The development of biotubes, in which vascular endothelial cells ${ }^{11}$ and bone marrow cells ${ }^{12}$ are seeded in artificial vascular grafts before implantation, has also been reported. ${ }^{13}$ The disadvantages of these grafts include the time to create the grafts and long-term preservation of the grafts. Compared with synthetic polymers, natural biopolymers, such as collagen, elastin, and silk fibroin (SF), offer better cytocompatibility and biocompatibility with embedded structural and functional molecules. ${ }^{14-16} \mathrm{SF}$ is one of the most preferred natural materials because of its superior mechanical properties compared with other biological materials. ${ }^{9}$

Our research group has conducted implant experiments using small-diameter artificial blood vessels made of SF. However, most of the implantations have been in the abdominal aorta of rats. ${ }^{17-20}$ According to the few studies focused on the long-term patency of small-diameter grafts in the carotid artery of dogs, no significant differences were observed in SF grafts compared with the existing ePTFE. ${ }^{21}$ Moreover, the endothelialization of the central part of the graft was not complete. ${ }^{22}$ One of the causes of poor patency in coated SF grafts in dogs is the delayed biodegradation and remodeling of SF grafts. Thus, instead of using alcohol, SF was mixed with glycerin (used as a porogen) as part of the insolubilization treatment of SF to develop a coating that is easily decomposed. ${ }^{23} \mathrm{SF}$ grafts coated with an amorphous SF sponge, prepared via glycerin removal, were implanted in the abdominal aortas of rats, and the remodeling ability was analyzed. The newly developed SF grafts were found to be more flexible, and the remodeling ability was greater than the conventional SF grafts insolubilized with alcohol. ${ }^{24}$ This study aimed to investigate the patency and remodeling ability of the new small-diameter SF artificial vascular grafts in a large animal model. Thus, we implanted SF grafts coated with amorphous SF sponge in the femoral arteries of dogs.

\section{METHODS}

\section{Preparation of the SF Vascular Grafts}

For the preparation of vascular SF grafts, SF double-raschel knit tubes of SF threads with an inner diameter of $3.5 \mathrm{~mm}$ and an outer diameter $4.0 \mathrm{~mm}$ were prepared by double barcode knitting of 30 gauges, 10 needles, and 20 courses on a computer-controlled double-raschel knit machine (Fukui Warp Knitting Co Ltd, Fukui City, Japan). ${ }^{21,22}$ It was necessary to use SF fibers with a small amount of silk sericin to maintain a degree of thread strength and avoid breaking SF threads in the knitting process. Therefore, the knit SF tube was degummed in a mixture of sodium carbonate $(0.08 \% \mathrm{w} / \mathrm{v})$ and Marseille soap $(0.12 \% \mathrm{w} / \mathrm{v})$ solution

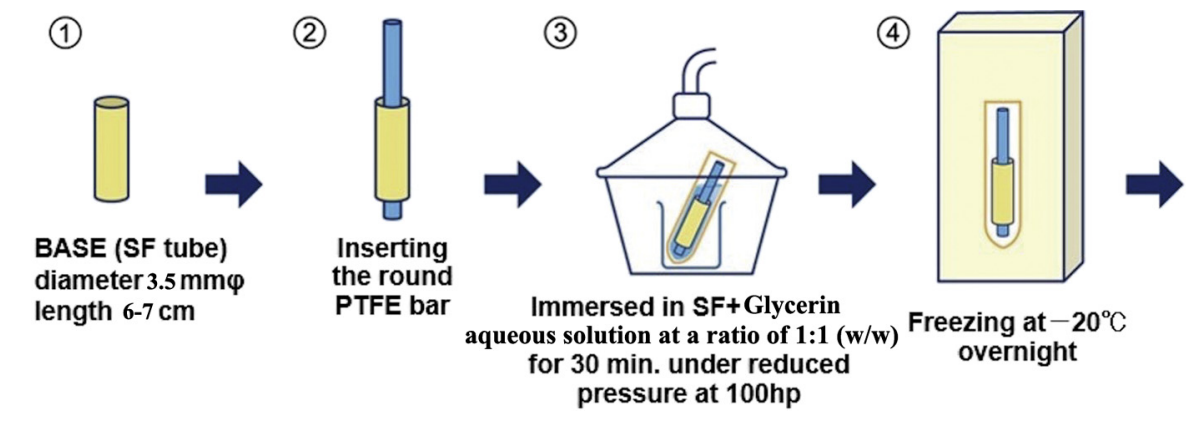

(5)

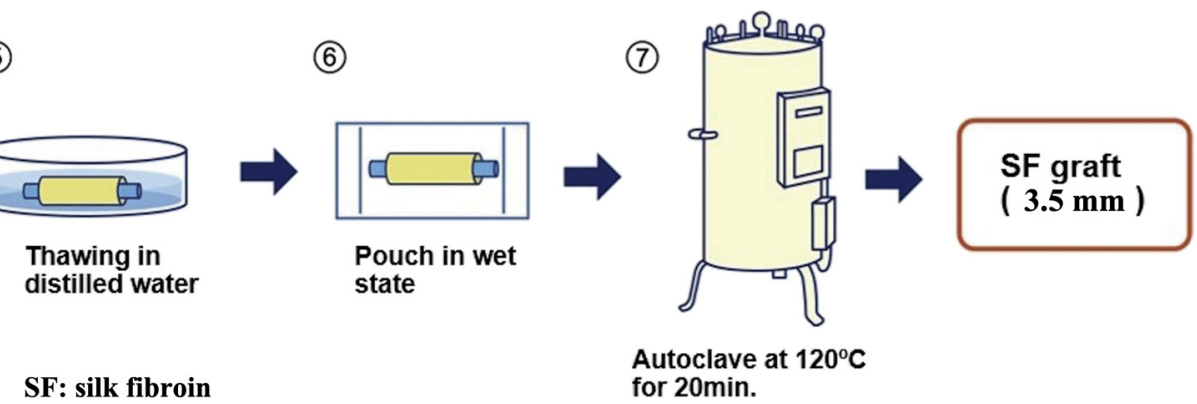

FIGURE 1. Process of preparation of a SF vascular graft coated by SF sponge using glycerin. SF, Silk fibroin; PTFE, polytetrafluoroethylene. 


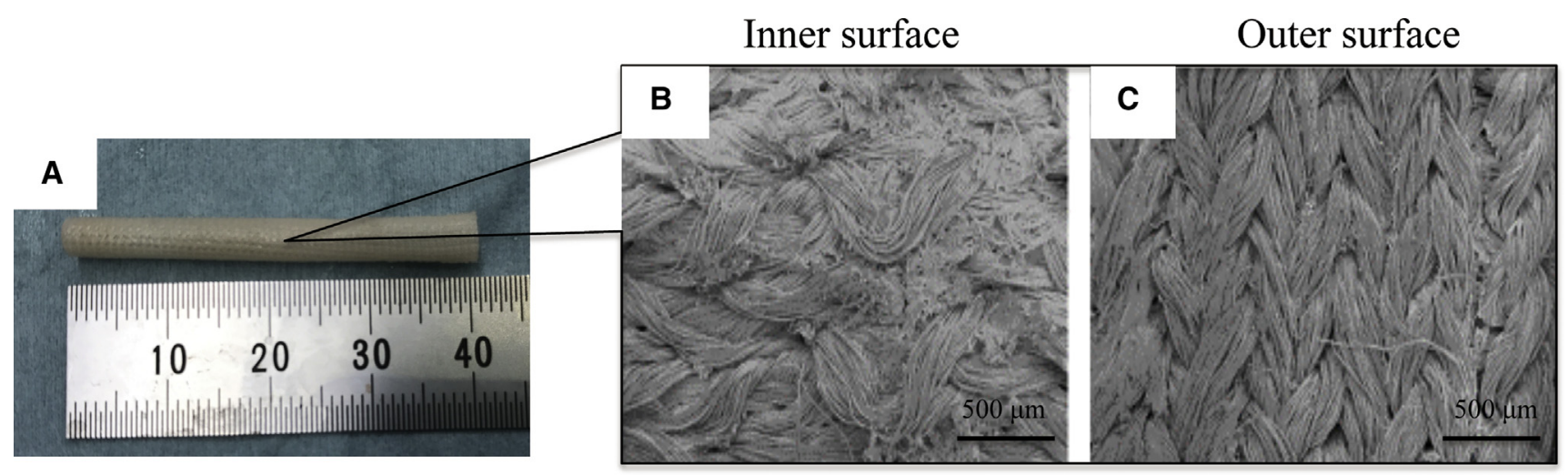

FIGURE 2. A, A 3.5-mm-inner-diameter and 4-cm-length artificial vascular graft was implanted in the femoral artery of a beagle. Scanning electron microscope pictures of the morphologies of the inner (B) and outer surfaces (C) of the silk fibroin vascular grafts.

at $95^{\circ} \mathrm{C}$ for 2 hours to remove the remaining silk sericin. Subsequently, a PTFE rod with a diameter of $3.5 \mathrm{~mm}$ was inserted into the knit SF tube. The rod covered with the SF tube was immersed into a pipe filled with a mixed aqueous solution of SF and glycerin (as a porogen) at a ratio of 1:1 (w/w) for coating. Preparation of the SF aqueous solution was described previously. ${ }^{17-24}$ The pipe was then placed in a desiccator under reduced pressure of $100 \mathrm{hPa}$ until no air bubbles were observed on the coated surface of the SF tube. The SF graft was then frozen at $-20^{\circ} \mathrm{C}$ overnight, immersed in distilled water for 3 days to remove the glycerin, and then bagged with distilled water. The pouch was sterilized in an autoclave at $120^{\circ} \mathrm{C}$ for 20 minutes (Figure 1). The SF graft was kept in distilled water until use. The morphology of the knit SF graft in the dry state was observed using a scanning electron microscope (VE7800; Keyence, Osaka, Japan) (Figure 2). The physical properties were determined using a tensile testing machine EZ graph (Shimadzu, Kyoto,
Japan) as previously reported. ${ }^{19,20,24}$ The longitudinal suture retention strength $(\mathrm{N})$, circumferential tensile strength $(\mathrm{N})$, and circumferential compressive elastic modulus $\left(\mathrm{N} / \mathrm{mm}^{2}\right)$ of the SF grafts were $6.4 \pm 0.6$, $51.0 \pm 3.0$, and $0.013 \pm 0.002$, respectively.

\section{Surgical Procedure}

SF grafts ( $4 \mathrm{~cm}$ length $\times 3.5 \mathrm{~mm}$ inner diameter) were implanted in either side of the femoral artery of 6 dogs under a stereoscopic microscope. Butorphanol (0.2 mg/kg; Meiji Seika Pharma Co, Ltd, Tokyo) and midazolam $(0.1 \mathrm{mg} / \mathrm{kg}$; Sandoz Ltd Novartis, Basel, Switzerland) were administered intravenously to each dog as a pre-anesthetic. In addition, ampicillin sodium ( $20 \mathrm{mg} / \mathrm{kg}$; Meiji Seika Pharma Co, Ltd) was administered intravenously before the skin was incised to prevent postoperative infection. Propofol (6 mg/kg; Fresenius Kabi Ltd, Bad Homburg, Germany) was also administered, and anesthesia was applied until
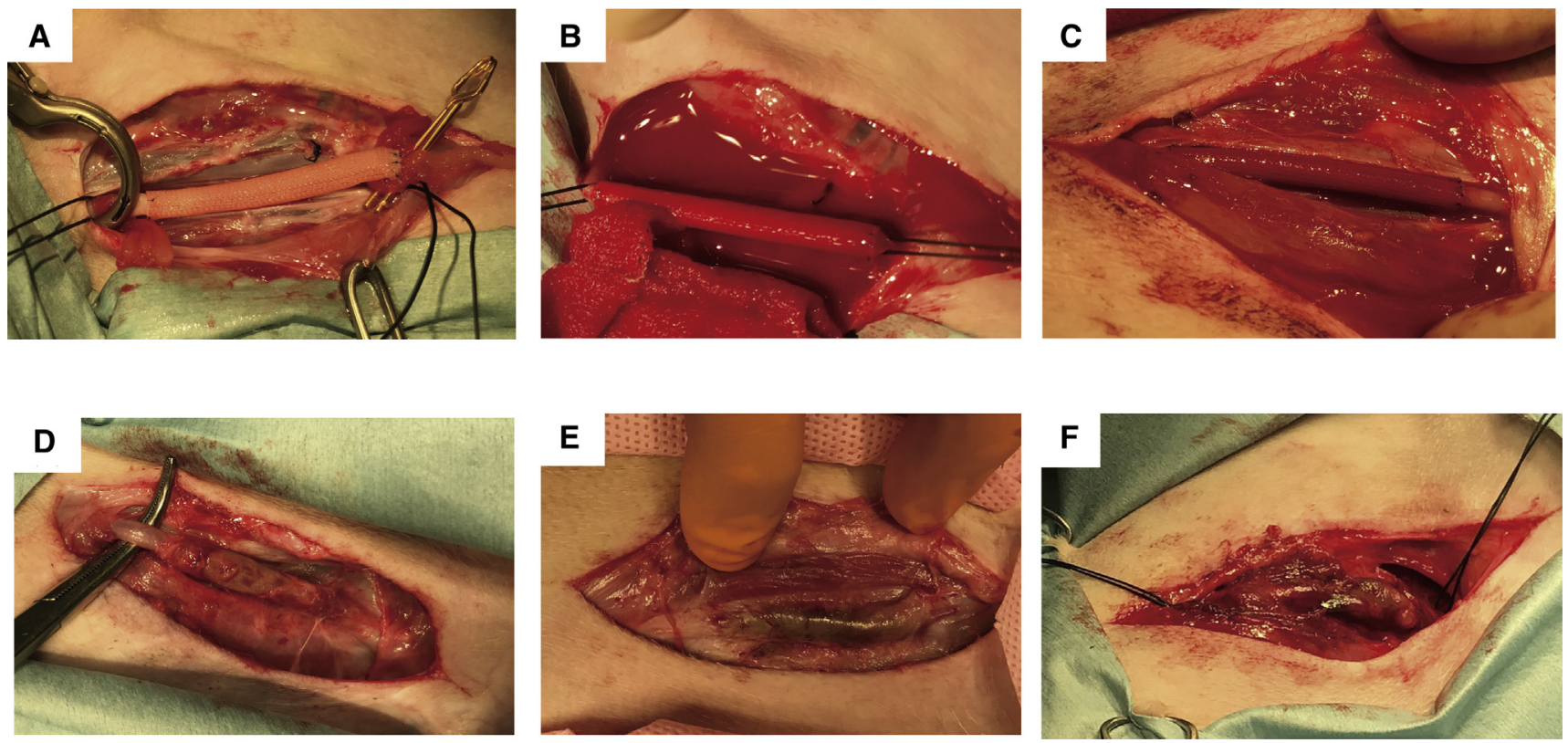

FIGURE 3. Photographs of artificial vascular grafts at the time of implantation. A, Artificial vascular grafts immediately after implantation in the femoral artery of a beagle; B, artificial vascular grafts after blood flow resumed; and C, artificial vascular grafts after hemostasis. A small amount of bleeding was observed in the graft, but it could be stopped by compression for several minutes. Photographs of vascular grafts at D, 3 months; E, 5 months; and F, 1 year postimplantation. The implanted vascular grafts adhered to the surrounding tissue and could be easily separated at all time points. 


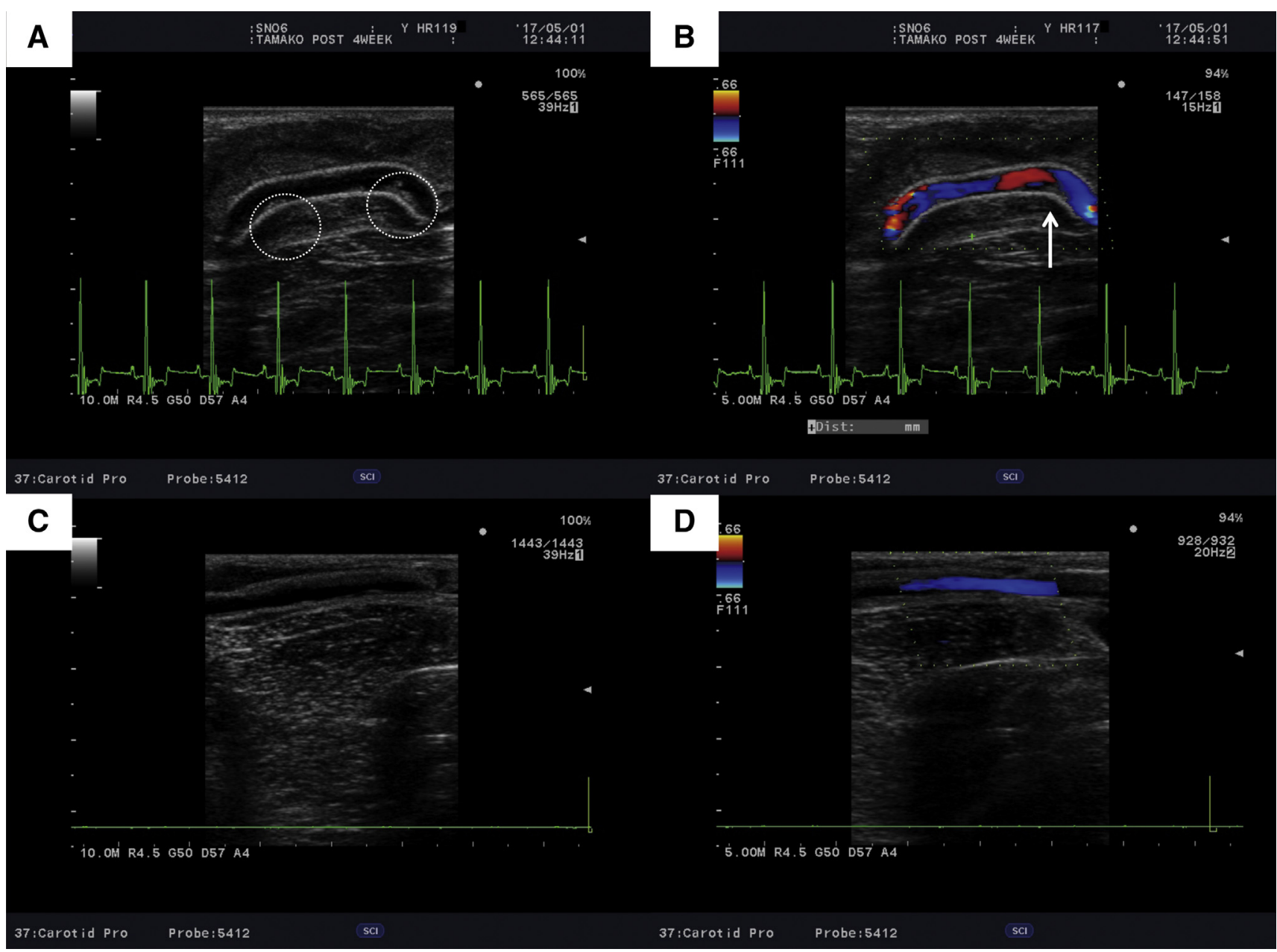

FIGURE 4. Vascular ultrasound examination of the implanted artificial vascular grafts. Vascular ultrasound photograph 4 weeks after transplantation. A structure with high echogenicity was confirmed on the lumen surface of the bent artificial vascular graft (A: circled). Blood flow could be confirmed via color Doppler. However, no blood flow was observed at the site of the thrombus (B: arrow). Vascular ultrasound photograph of artificial vascular graft 1 year postimplantation (C). No bending or aneurysm of the artificial vascular graft was observed. D, In addition, the blood flow was confirmed using a color Doppler scan.

endotracheal intubation. Respiration was maintained using a respirator via an orotracheal tube, and the dogs were ventilated with a mixture of oxygen and isoflurane. The femoral arteries were carefully exposed, and the aortic branches in this segment were ligated. After intravenous heparin injection (100 IU/kg; AY Pharmaceuticals Ltd, Japan), the proximal and distal portions of the femoral arteries were clamped using noncrushing vascular clamps. SF grafts were implanted in the femoral arteries via end-to-end anastomoses using 7-0 monofilament polyvinylidene fluoride sutures (Peters Surgical Ltd, Haryana, India). The distal and proximal vascular clamps were slowly removed, and the flow was restored through the grafts. Amoxicillin (PASETOCIN 250 tablets; Aspen Japan Ltd) was orally administered twice a day for 1 week after the operation for concerns about self-licking and postoperative contamination. In addition, clopidogrel (TEVA 25 Tablets; Teva Takeda Pharma Ltd, Nagoya, Japan) was administered orally once a day until the graft was removed. The patency of the graft was monitored via color Doppler imaging (e-flow imaging). Moreover, the abnormalities in the graft cavity were investigated using a 7.5$\mathrm{MHz}$ linear probe and vascular ultrasound apparatus (Prosound a-10; Hitachi-Aloka, Twinsburg, Ohio) at 1 day after surgery; then at 1, 2, 3, and 4 weeks after surgery, and finally every month until removal. Data are presented as mean and standard error. Comparison of means was performed by one-way analysis of variance. Data analysis was performed using the commercial statistics software package GraphPad Prism (Version 5.0a, San Diego, Calif).

At 3 months, 5 months, and 1 year postimplantation, general anesthesia was applied via the same procedure as the surgery, and the graft was retrieved. All experimental procedures and protocols were approved by the Tokyo University of Agriculture and Technology (TUAT, approval number: 29-38). The dogs were managed and cared for in accordance with the standards established by Tokyo University of Agriculture and Technology and described in its "Guide for the Care and Use of Laboratory Animals."

\section{Histopathologic Examination}

After the removal of the graft, it was immersed in $99.5 \%$ methanol for one night. A 4-cm-long section of the artificial vascular graft, which was 4-mm wide and cut in the short-axis direction, was immersed in $99.5 \%$ methanol for 30 minutes, dehydrated, immersed in xylene for 90 minutes, and then embedded in paraffin. The embedded samples were sliced in 5- $\mu \mathrm{m}$ sections. The prepared paraffin sections were subjected to hematoxylin and 

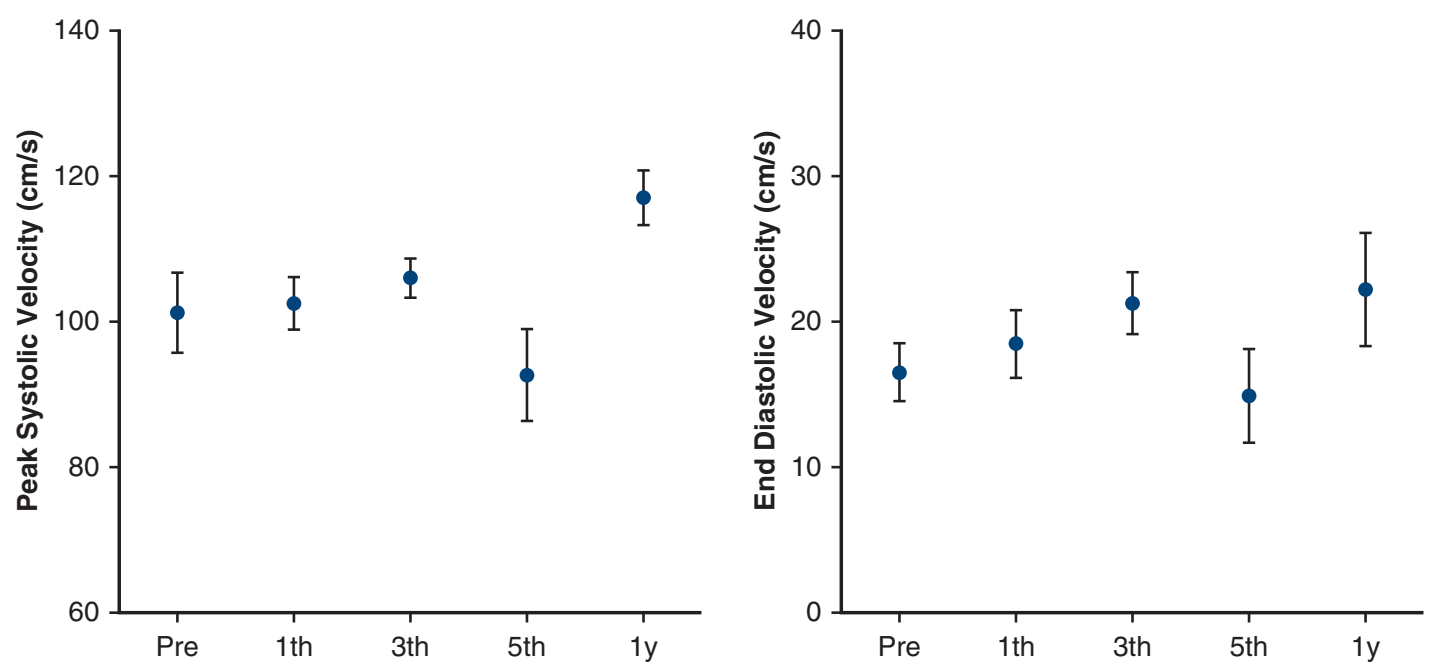

FIGURE 5. Peak systolic velocity and end-diastolic velocity values of the central part of the graft postimplantation. No significant change was observed in all postimplant periods. Peak systolic velocity preimplantation was $101.25 \pm 12.31$, 1-month postimplantation was $102.51 \pm 8.04,3$-month postimplantation was $106.04 \pm 5.31,5$-month postimplantation was $92.63 \pm 8.88$, and 1-year postimplantation was $117.05 \pm 3.75$. End-diastolic velocity preimplantation was $16.51 \pm 4.46,1$-month postimplantation was $18.46 \pm 5.164$, 3-month postimplantation was $21.28 \pm 4.25$, 5-month postimplantation was $14.9 \pm 4.52$, and 1-year postimplantation was $22.2 \pm 3.9$.

eosin, Masson's trichrome, and Elastica van Gieson staining according to the standard procedures. ${ }^{20,22}$ For immunohistochemical staining, $\alpha$-smooth muscle actin mouse anti-monoclonal antibody (Sigma-Aldrich Inc, St Louis, Mo) and CD31 anti-rabbit polyclonal antibody (Abcam Inc, Cambridge, Mass) were used as primary antibodies, followed by anti-mouse IgG biotin (Nichirei Biosciences Inc, Tokyo, Japan) secondary antibodies. Vectastain ABC-AP (Vector laboratories Inc, Burlingame, Calif) was used for color development. By using these staining methods, the ability to remodel autologous tissue postimplantation, ie, replacement with vascular endothelial cells, smooth muscle cells, elastic fibers and collagen fibers, intima thickening with narrowing of the lumen, and inflammatory reaction, was investigated.

\section{RESULTS}

\section{In Vivo Experiments}

Six beagles weighing 10 to $12 \mathrm{~kg}$ were used for the in vivo study. All 6 implantations were successfully performed with no complications, such as uncontrolled bleeding. Although mild bleeding was observed in the grafts and anastomotic sites, it was stopped by compression with a cotton swab for several minutes. No side effects, such as paralysis and hematoma, were observed. In addition, no changes in the degree of adhesion between grafts and the surrounding tissues developed at 3 months, 5 months, and 1 -year postimplantation. The grafts seemed to have adhered to the surrounding muscles by thin fibrous tissue (Figure 3). No aneurysms or infections were observed. However, in one case, occlusion due to thrombus formation was observed 4 weeks' postimplantation.

\section{Ultrasonic Examination}

All small-diameter SF artificial vascular grafts were evaluated postimplantation using an ultrasonic examination apparatus. No acute side effects were observed, such as thrombus formation in the lumen of the graft immediately postimplantation or hematoma formation due to blood leakage. In one case, sudden bending occurred in the lateral direction of the trunk on both the proximal and distal sides of the graft 2 weeks postimplantation, and the angle of the curve gradually increased with time. A thrombus was formed at the bent site, and the graft was confirmed to be obstructed 4 weeks postimplantation (Figure 4). Several high echogenic structures, which were not detected by color Doppler, were observed in the lumen of the graft. In the remaining 5 cases, the side effects described above were not observed at 3 months, 5 months, and 1 year. There was also no intimal hyperplasia or aneurysms. Hemodynamic changes, including peak systolic velocity and enddiastolic velocity, were determined. No significant changes in peak systolic velocity and end-diastolic velocity in the central part of the graft were observed (Figure 5).

\section{Histopathologic Examination}

A layered structure was identified along the luminal surface of the graft using hematoxylin and eosin staining. The thickness increased at the 5-months and 1-year time points, compared with 3 months' postimplantation (Figure 6). The layered structure was mainly composed of smooth muscle cells, elastic fibers, and collagen fibers, and no difference was observed in the constituent components during each implantation period. Inflammatory cells, such as neutrophils, lymphocytes, and macrophages, were concentrated around the graft but could not be found around the base of the graft or in the lumen at 3 months postimplantation. 

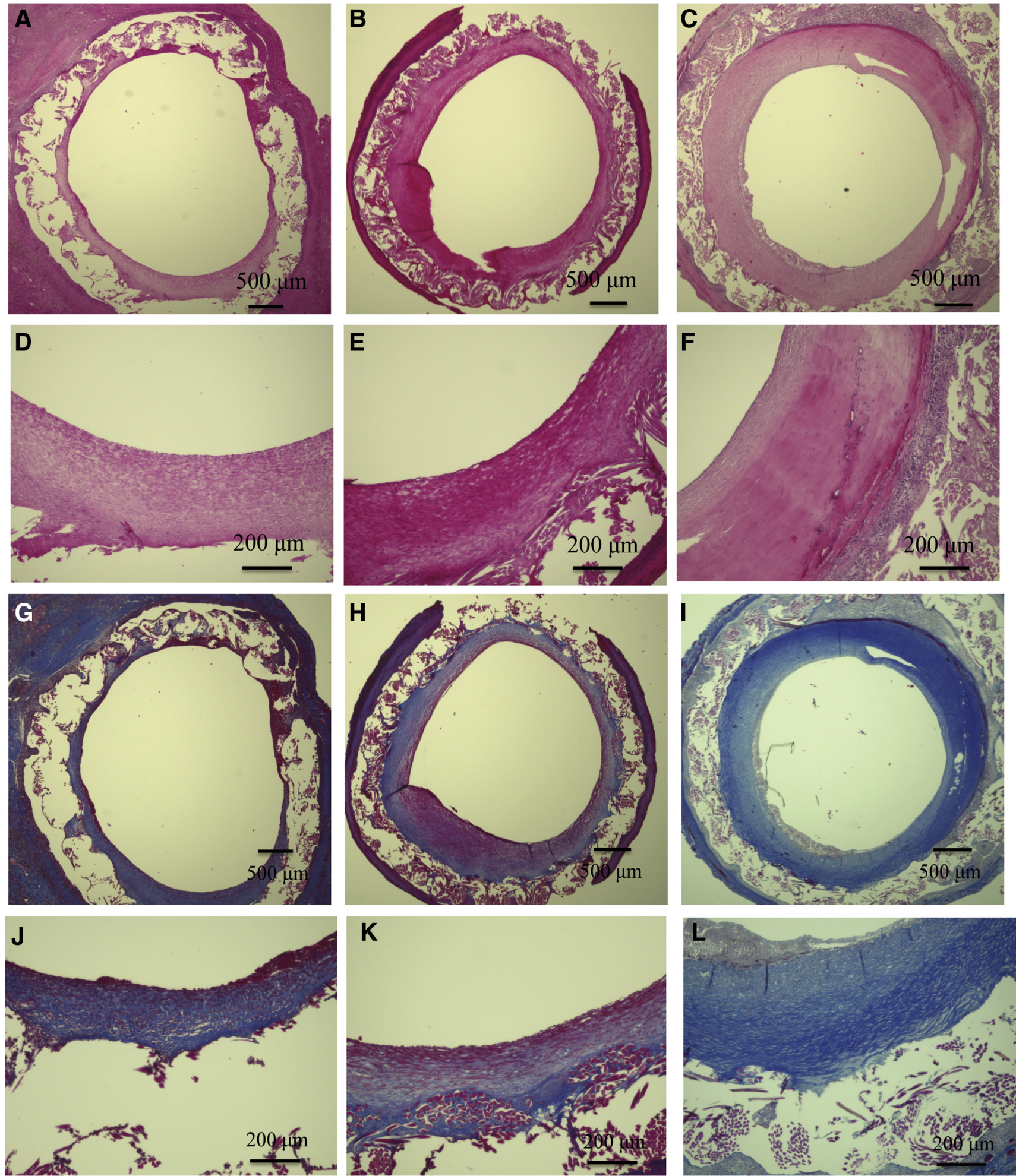

FIGURE 6. HE and MTC staining 3 months, 5 months, and 1-year postimplantation. HE staining at 3 months' postimplantation (A) and its highmagnification image (D), HE staining at 5 months' postimplantation (B) and its high-magnification image (E), HE staining at 1-year postimplantation (C) and its high-magnification image (F), MTC staining at 3 months' postimplantation (G) and its high-magnification image (J), MTC staining at 5 months' postimplantation $(\mathrm{H})$ and its high-magnification image (K), and MTC staining at 1-year postimplantation (I) and its high-magnification image (L) are presented. Layered structures formed in the lumen of the artificial vascular graft at 3 months' postimplantation, and their thickness increased 5 months and 1 -year postimplantation. Collagen fibers were mainly gathered around the outer periphery of the artificial vascular graft 3 months' postimplantation. At 5 months and 1-year postimplantation, the collagen fibers on the luminal surface increased, and the collagen fibers on the outer peripheral surface decreased. 
The inflammatory cells decreased with the prolongation of the transplantation period and significantly decreased in the periphery of the graft 5 months' postimplantation. Similarly, about 3 months' postimplantation, numerous collagen fibers were observed on the outer periphery and the luminal surface of the graft. However, the thickness range was uneven. At 5 months and 1-year postimplantation, the quantity of collagen fibers accumulated in the lumen of the graft was increased. Conversely, the quantity of collagen fibers accumulated at the outer periphery of the graft decreased. Smooth muscle cells and elastic fiber layers were observed in the lumen of the central part of the graft 3 months postimplantation, and vascular endothelial cells were found in the innermost surface of the graft (Figure 7). These structures did not change at 5 months and 1 year postimplantation compared with the 3-month time point. Granulomas, aneurysms, and calcification were not observed in the remodeled blood vessels.

\section{DISCUSSION}

Small-diameter artificial vascular grafts using SF have been investigated, and the useful characteristics of SF have been demonstrated. ${ }^{17-22,24}$ However, there have been only a few reports of experiments in large animal models due to the limitations of graft length, number of transplants, and short transplantation periods compared with small animal models. Although vascular prostheses made of SF exhibited high patency and remodeling potential in vivo, they have all been evaluated in the abdominal aorta of rats. ${ }^{25-27}$ The challenge was to evaluate artificial vascular grafts made of SF in vivo in larger animal models. ${ }^{28}$ In a few studies, artificial vascular grafts made of SF in vivo in dogs were evaluated. Aytemiz and colleagues implanted vascular grafts with a diameter of $3 \mathrm{~mm}$ in the carotid arteries of dogs and evaluated the grafts in vivo. In this study, 1 of 5 implants was patent for more than a year. However, the endothelialization of the graft lumen was incomplete. ${ }^{22}$ Similarly, Haga and colleagues ${ }^{21}$ implanted SF vascular grafts in the carotid arteries of dogs to examine the patency and compared the SF grafts with the ePTFE grafts. However, the patency rates of the SF and the ePTFE grafts at 6 months postimplantation were $7.8 \%$ and $0 \%$, respectively. No significant differences were observed in the patency rate between the SF and ePTFE vascular grafts. Dogs are more susceptible to blood clotting than other animal species ${ }^{29}$; thus, clotting could not be prevented postimplantation of SF vascular grafts.

SF promotes endogenous tissue migration and antithrombotic effects and has been applied in tissue engineering. ${ }^{30}$ However, the SF coating around the vascular graft, which decomposes slowly, is not optimal. ${ }^{23}$ Therefore, we improved the coating method to produce an SF vascular graft that can be easily degraded and remodeled with endogenous cells in vivo postimplantation. Thus far, all in vivo evaluations have been conducted on the abdominal aorta of rats. ${ }^{24}$ In this study, we examined whether the coating method developed in this study could increase the patency rate and improve the remodeling ability in an animal model larger than the rat.

In this study, the optimal physical properties of the artificial vascular graft were not determined as the same manufacturing methods were applied as in a previous study. ${ }^{24}$ However, new small-diameter SF artificial vascular grafts showed almost the same physical property values as previous SF and ePTFE grafts. ${ }^{21}$ Similar to the previous study, the artificial vascular graft was not ruptured by suture, and no aneurysms developed postimplantation. Therefore, even though the diameter was increased to $3.5 \mathrm{~mm}$, the graft exhibited sufficient strength for implantation. When the vascular clamps were removed and the blood flow resumed postimplantation, blood leakage was observed. The 3.5-mm-diameter graft leaked more blood than the
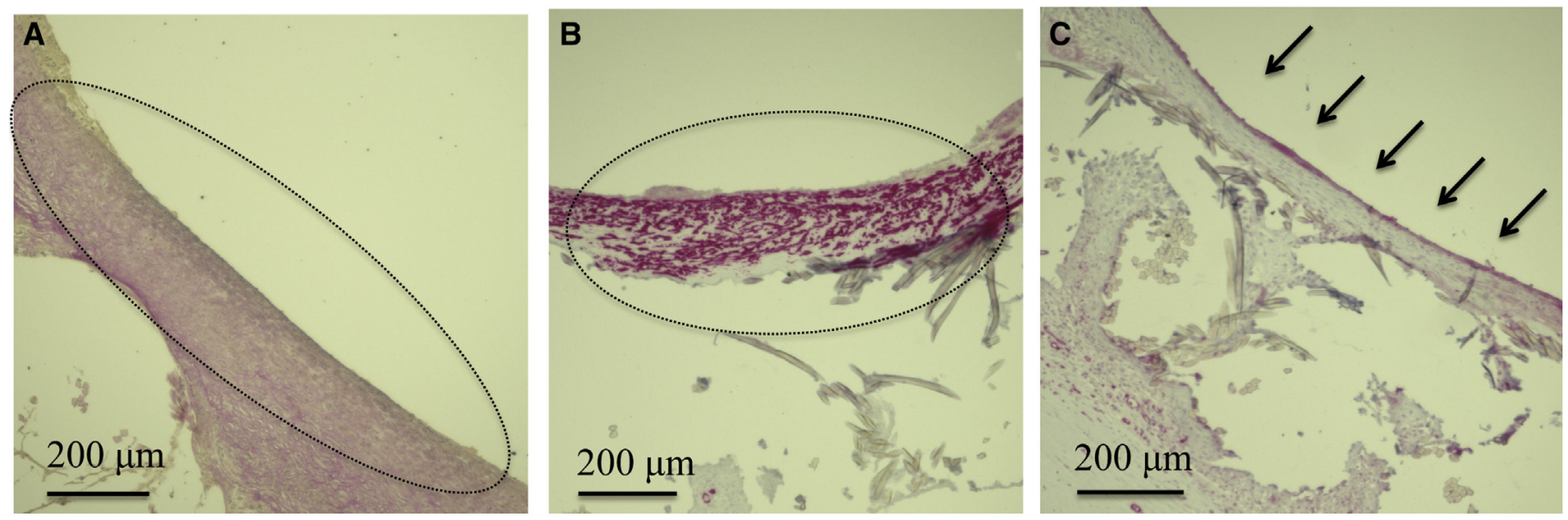

FIGURE 7. Photographs at 3 months' postimplantation. A, Elastica van Gieson staining, B, $\alpha$-smooth muscle actin staining, and C, CD31 staining are presented. Elastic fibers, smooth muscle cells, and endothelial cells were observed in the intimal layer of artificial vascular grafts and their surfaces. 
SF grafts implanted in rats due to the larger mesh of the base. However, even during anticoagulation treatment with heparin, implantation was possible in all cases using only mild compression with gauze to stop the bleeding. A greater coating concentration is thought to make vascular grafts harder and easier to implant, but the degradation of SF is delayed in vivo and remodeling is impeded. ${ }^{20}$ In this study, the improved SF coating was rapidly degraded postimplantation. Therefore, coating at a concentration greater than the conventional optimal SF coating concentration had no effect on the remodeling ability postimplantation. Thus, the optimal SF coating concentration in SF artificial vascular grafts with a diameter of $3.5 \mathrm{~mm}$ was reconsidered.

In this study, the implanted vascular graft exhibited a patency rate of more than $80 \%$ postimplantation. At 3 months postimplantation, remodeling to the autologous tissue was observed to be similar to the implantations in rats. As the length of the postimplantation period increased, the thickness of the intima gradually increased, but no lumen narrowing was observed. The vascular endothelial cells are thought to prevent vascular lumen stenosis due to blood coagulation and excessive proliferation of smooth muscle cells. ${ }^{31}$ Therefore, even if the course of transplantation was longer than 1 year, intimal thickening should be prevented once the vascular endothelial cells covered the lumen of the artificial vascular graft. In addition, rupture, aneurysm, and calcification were not observed even 1 year postimplantation, thus indicating that high patency will be maintained for a long term.

In 1 of the 6 cases, the vascular graft was bent postimplantation. The artificial blood vessel was bent 2 weeks postimplantation, and the angle of the curve gradually increased with time. Turbulent blood flow occurred at the implantation site, and a thrombus formed, which resulted in occlusion. Neointimal formation in transplanted vascular grafts involves (1) endothelialization by stretching from the anastomotic site, (2) endothelialization from capillaries extending through the gap between the artificial vascular graft fibers, and (3) endothelialization by adhesion of vascular endothelial progenitor cells circulating in the blood. ${ }^{32}$ In this study, the application of an easily degradable coating to the artificial vascular graft facilitated faster biodegradation. At the same time, the capillary blood vessel and collagen fibers passed through gaps in the base. Thus, endothelialization of the graft was faster. As histologic examination was not conducted 4 weeks postimplantation, the revascularization status of the vascular graft was unknown. Endothelial cells may not have completely covered the luminal surface of the grafts at 4 weeks. Therefore, once an event like bending of the artificial vascular graft occurred, the graft could have easily become occluded. Thus, endothelialization in the central part of the transplanted artificial vascular graft needs to be further improved. SF can be genetically modified via the introduction of new functions into silkworm genes. ${ }^{33}$ With this technology, it is possible to accelerate endothelialization in artificial vascular grafts by incorporating vascular endothelial growth factor, a factor that promotes the growth of endothelial cells, into the base $\mathrm{SF}^{34}$ In additions, for application in human medicine, the length of the artificial vascular graft needs to be further increased. ${ }^{35}$ If the artificial vascular graft is lengthened, it is possible that the incidence of bending of the grafts will increase postimplantation. Therefore, we plan to investigate whether high patency and remodeling ability can be exhibited even at clinically applicable lengths.

\section{LIMITATIONS}

There were 3 limitations to this study. First, there was no control group in this study. Second, antiplatelet drug was being administered until graft removal. Third, the implantation of artificial SF vascular grafts in the femoral artery of dogs is preliminary model. In future study, while increasing the number of implanted SF grafts in large animal model, we will investigate whether it is possible to maintain patency without using antiplatelet drugs postimplantation, and whether it is superior in remodeling ability and biocompatibility compared to commercially available artificial vascular grafts.

\section{CONCLUSIONS}

For the clinical application of SF artificial vascular grafts, it was important to evaluate the implantation in larger animals. In this study, artificial SF vascular grafts implanted in the femoral arteries of dogs exhibit high patency and remodeling ability. SF grafts may be clinically applicable as an artificial vascular graft in small-diameter $<6 \mathrm{~mm}$.

\section{Conflict of Interest Statement}

The authors reported no conflicts of interest.

The Journal policy requires editors and reviewers to disclose conflicts of interest and to decline handling or reviewing manuscripts for which they may have a conflict of interest. The editors and reviewers of this article have no conflicts of interest.

\section{References}

1. Pashneh-Tala S, MacNeil S, Claeyssens F. The tissue-engineered vascular graft_-past, present, and future. Tissue Eng Part B Rev. 2016;22:68-100.

2. Budd JS, Allen KE, Hartley G, Bell PRF. The effect of preformed confluent endothelial cell monolayers on the patency and thrombogenicity of small calibre vascular grafts. Eur J Vasc Surg. 1991;5:397-405.

3. Takagi H, Goto S, Matsui M, Manabe H, Umemoto T. A contemporary metaanalysis of Dacron versus polytetrafluoroethylene grafts for femoropopliteal bypass grafting. J Vasc Surg. 2010;52:232-6.

4. Ballyk PD, Walsh C, Butany J, Ojha M. Compliance mismatch may promote graft-artery intimal hyperplasia by altering suture-line stresses. J Biomech 1998;31:229-37.

5. Haruguchi H, Teraoka S. Intimal hyperplasia and hemodynamic factors in arterial bypass and arteriovenous grafts: a review. J Artif Organs. 2003;6:227-35. 
6. Pektok E, Nottelet B, Tille J-C, Gurny R, Kalangos A, Moeller M, et al. Degradation and healing characteristics of small-diameter poly(epsilon-caprolactone) vascular grafts in the rat systemic arterial circulation. Circulation. 2008;118: 2563-70.

7. Jing X, Mi H-Y, Salick MR, Cordie TM, Peng X-F, Turng L-S. Electrospinning thermoplastic polyurethane/graphene oxide scaffolds for small diameter vascular graft applications. Mater Sci Eng C. 2015;49:40-50.

8. Wang W, Hu J, He C, Nie W, Feng W, Qiu K, et al. Heparinized PLLA/PLCL nanofibrous scaffold for potential engineering of small-diameter blood vessel: tunable elasticity and anticoagulation property. J Biomed Mater Res A. 2015; 103:1784-97.

9. Wang D, Liu H, Fan Y. Silk fibroin for vascular regeneration. Microsc Res Tech. 2017;80:280-90.

10. Naito Y, Shinoka T, Duncan D, Hibino N, Solomon D, Cleary M, et al. Vascular tissue engineering: towards the next generation vascular grafts. Adv Drug Deliv Rev. 2011;63:312-23.

11. Laube HR, Duwe J, Rutsch W, Konertz W. Clinical experience with autologous endothelial cell-seeded polytetrafluoroethylene coronary artery bypass grafts. $J$ Thorac Cardiovasc Surg. 2000;120:134-41.

12. Roth GA, Forouzanfar MH, Moran AE, Barber R, Nguyen G, Feigin VL, et al. Demographic and epidemiologic drivers of global cardiovascular mortality. $N$ Engl J Med. 2015;372:1333-41.

13. Ma N, Wang Z, Chen H, Sun Y, Hong H, Sun Q, et al. Development of the novel biotube inserting technique for acceleration of thick-walled autologous tissueengineered vascular grafts fabrication. J Mater Sci Mater Med. 2011;22:1037-43.

14. Stamati K, Priestley JV, Mudera V, Cheema U. Laminin promotes vascular network formation in 3D in vitro collagen scaffolds by regulating VEGF uptake. Exp Cell Res. 2014;327:68-77.

15. Zhu C, Fan D, Wang Y. Human-like collagen/hyaluronic acid 3D scaffolds for vascular tissue engineering. Mater Sci Eng C. 2014;34:393-401.

16. Zhu M, Wang K, Mei J, Li C, Zhang J, Zheng W, et al. Fabrication of highly interconnected porous silk fibroin scaffolds for potential use as vascular grafts. Acta Biomater. 2014;10:2014-23.

17. Enomoto S, Sumi M, Kajimoto K, Nakazawa Y, Takahashi R, Takabayashi C, et al. Long-term patency of small-diameter vascular graft made from fibroin, a silk-based biodegradable material. J Vasc Surg. 2010;51:155-64.

18. Yagi T, Sato M, Nakazawa Y, Tanaka K, Sata M, Itoh K, et al. Preparation of double-raschel knitted silk vascular grafts and evaluation of short-term function in a rat abdominal aorta. J Artif Organs. 2011;14:89-99.

19. Fukayama T, Takagi K, Tanaka R, Hatakeyama Y, Aytemiz D, Suzuki Y, et al. Biological reaction to small-diameter vascular grafts made of silk fibroin implanted in the abdominal aortae of rats. Ann Vasc Surg. 2015;29:341-52.

20. Fukayama T, Ozai Y, Shimokawadoko H, Aytemiz D, Tanaka R, Machida N, et al. Effect of fibroin sponge coating on in vivo performance of knitted silk small diameter vascular grafts. Organogenesis. 2015;11:137-51.

21. Haga M, Yamamoto S, Okamoto H, Hoshina K, Asakura T, Watanabe T. Histological reactions and the in vivo patency rates of small silk vascular grafts in a canine model. Ann Vasc Dis. 2017;10:132-8.
22. Aytemiz D, Sakiyama W, Suzuki Y, Nakaizumi N, Tanaka R, Ogawa Y, et al. Small-diameter silk vascular grafts (3 $\mathrm{mm}$ diameter) with a double-raschel knitted silk tube coated with silk fibroin sponge. Adv Healthc Mater. 2013;2: 361-8.

23. Asakura T, Endo M, Fukuhara R, Tasei Y. ${ }^{13} \mathrm{C}$ NMR characterization of hydrated 13C labeled Bombyx mori silk fibroin sponges prepared using glycerin, poly(ethylene glycol diglycidyl ether) and poly(ethylene glycol) as porogens. J Mater Chem B. 2017;5:2152-60.

24. Tanaka T, Uemura A, Tanaka R, Tasei Y, Asakura T. Comparison of the knitted silk vascular grafts coated with fibroin sponges prepared using glycerin, poly(ethylene glycol diglycidyl ether) and poly(ethylene glycol) as porogens. J Biomater Appl. 2018;32:1239-52.

25. Cattaneo I, Figliuzzi M, Azzollini N, Catto V, Farè S, Tanzi MC, et al. In vivo regeneration of elastic lamina on fibroin biodegradable vascular scaffold. Int $J$ Artif Organs. 2013;36:166-74.

26. Filipe EC, Santos M, Hung J, Lee BSL, Yang N, Chan AHP, et al. Rapid endothelialization of off-the-shelf small diameter silk vascular grafts. JACC Basic Transl Sci. 2018;3:38-53.

27. Lovett M, Eng G, Kluge J, Cannizzaro C, Vunjak-Novakovic G, Kaplan DL. Tubular silk scaffolds for small diameter vascular grafts. Organogenesis. 2010; 6:217-24.

28. Holland C, Numata K, Rnjak-Kovacina J, Seib FP. The biomedical use of silk: past, present, future. Adv Healthc Mater. 2019;8:1800465.

29. Sato M, Harasaki H. Evaluation of platelet and coagulation function in different animal species using the xylum clot signature analyzer. ASAIO J. 2002; $48: 360-4$

30. Thurber AE, Omenetto FG, Kaplan DL. In vivo bioresponses to silk proteins. Biomaterials. 2015;71:145-57.

31. Tai NR, Salacinski HJ, Edwards A, Hamilton G, Seifalian AM. Compliance properties of conduits used in vascular reconstruction. Br J Surg. 2000;87: 1516-24.

32. Noishiki Y, Yamane Y, Tomizawa Y, Matsumoto A. Transplantation of autologous tissue fragments into an e-PTFE graft with long fibrils. Artif Organs. 1995; 19:17-26.

33. Inoue S, Kanda T, Imamura M, Quan G-X, Kojima K, Tanaka H, et al. A fibroin secretion-deficient silkworm mutant, Nd-sD, provides an efficient system for producing recombinant proteins. Insect Biochem Mol Biol. 2005;35:51-9.

34. Saotome T, Hayashi H, Tanaka R, Kinugasa A, Uesugi S, Tatematsu K, et al. Introduction of VEGF or RGD sequences improves revascularization properties of Bombyx mori silk fibroin produced by transgenic silkworm. J Mater Chem B. 2015;3:7109-16.

35. Mahara A, Somekawa S, Kobayashi N, Hirano Y, Kimura Y, Fujisato T, et al. Tissue-engineered acellular small diameter long-bypass grafts with neointimainducing activity. Biomaterials. 2015;58:54-62.

Key Words: small-diameter vascular grafts, silk fibroin, remodeling ability, endothelialization, large animal model 